\title{
Heterotopic ossification and rhabdomyolysis
}

\author{
F Béthoux ${ }^{1}$, P Calmels ${ }^{1}$, JL Aigoin ${ }^{2}$, FG Barral ${ }^{2}$, M Domenach $^{1}$ and P Minaire ${ }^{1}$ \\ ${ }^{1}$ Services de Rééducation et Réédaptation Fonctionnelles, Hôpital Bellevue, 42055 Saint Etienne Cedex 2 and GIP \\ Exercice, Université Jean Monnet, 42 Saint Etienne; ${ }^{2}$ Service Central de Radiologie, Hôpital Bellevue, 42055 \\ Saint Etienne Cedex 2, France
}

\begin{abstract}
Heterotopic ossification and rhabdomyolysis are well described entities but, as far as we know, their association has never been described in the literature. We recently treated a patient who presented with this association. After a suicide attempt, this patient developed rhabdomyolysis of the left upper and lower limbs with peripheral neurological impairment. Two months later radiographs showed ectopic ossification around the left hip. Rhabdomyolysis is underdiagnosed, and is due to local disturbance of the calcium-phosphorus metabolism resulting in soft tissue calcifications. Underlying rhabdomyolysis may be a possible aetiology of heterotopic ossification. Recognition of this may help us to understand the pathophysiology and to improve the management of heterotopic ossification.
\end{abstract}

Keywords: heterotopic ossification; rhabdomyolysis; calcium-phosphorus metabolism; neurological impairment

\section{Introduction}

Heterotopic ossification and rhabdomyolysis are wellknown entities, though their pathogenesis remains to be elucidated. To our knowledge, their association has never been reported in the literature. One of our patients presented with this association. This case leads us to suggest that rhabdomyolysis is an underdiagnosed cause of heterotopic ossification.

Heterotopic ossifications ${ }^{1-3}$ are ossifications of periarticular soft tissues, due to bone metaplasia of the connective tissue. The usual clinical signs are local inflammation, reduced joint motion and a slight rise in body temperature. The main complications are joint ankylosis and vascular and neural compression. The first radiological signs, which appear 4 to 10 days later, are: periarticular blur, hazy opacity of increasing density and a gradual delineation of heterotopic ossification. Bone scintigraphy with $99 \mathrm{mTc}$ pyrophosphate reveals early uptake. Quantitative scintigraphy allows assessment of bone maturation. ${ }^{4}$ On ultrasound examination, the normal lamellar pattern disappears. ${ }^{5} \mathrm{Com}$ puted tomography (CT), and MRI allow the relationship between heterotopic ossification and bone to be established.

Biochemically, a rise in blood alkaline phosphatases and hydroxyprolinuria (more precisely the nondialysable fraction of hydroxyproline) have been reported, as well as an initial peak of serum creatine phosphokinase (CPK). Calcium and phosphorus plasma levels are normal. Heterotopic ossifications are usually seen following lesions of the central nervous system (traumatic coma, toxic coma, hemiplegia, spinal cord injury, etc), but have also been described in patients with such conditions as polyneuritis, tetanus, burns, and after total hip prosthesis. The pathogenesis is still not fully elucidated, but several factors have been identified, ${ }^{2,3,6}$ including immobilisation, prolonged pressure exerted on periarticular structures, microtrauma (notably during rehabilitation), ${ }^{7,8}$ vascular stasis, lesions of the autonomic nervous system, hypoxia, hyperthermia, mobilisation of the calcium pool and genetic predisposition (groups HLA B 18 and DRW 7). ${ }^{9}$

Rhabdomyolysis ${ }^{10}$ is a syndrome secondary to the lysis of striated muscle cells, with release of their content into the extracellular space. The clinical signs are myalgia, muscle oedema, dark urine, and skin rashes.

The most serious complication is acute renal failure due to acute tubular necrosis with initial oliguria or anuria, then diuresis in 10-15 days with polyuria. There are also reports of vascular or neural compression, secondary to the muscular oedema, which may be very considerable. Calcium deposits have been observed in the soft tissues, detectable by scintigraphy ${ }^{11}$ and $\mathrm{CT} .{ }^{12}$ Such calcification, often reversible, can be observed either during the early oliguric phase or later during the polyuric phase.

Simple radiographs show a haziness of fat planes in muscle. On ultrasonography, ${ }^{13}$ the normal muscular echostructure disappears and is replaced by more or less well defined heterogeneous zones. Computed tomography ${ }^{14}$ reveals oedematous muscles followed by atrophy. MRI may also be helpful. It is important to note the presence of the increased uptake of the muscles concerned on scintigraphy in the first stages of the disease. ${ }^{15,16}$

The most characteristic biological sign is the rise in serum CPK, sometimes exceeding $5000 \mathrm{IUl}^{-1}$. Myoglobinemia and myoglobinuria are also of diagnostic value. The other muscular enzymes are increased. 
Cytolysis is also responsible for a large number of ionic disorders and metabolic disturbances. Disturbances of the calcium-phosphorus metabolism are often detected, particularly if there is acute renal failure ( $\mathrm{Cf}$ infra).

There are many aetiological factors involved in rhabdomyolysis: for example, crush syndrome, stress rhabdomyolysis, vascular occlusions, burns, toxic coma, malignant hyperthermia, infections, myopathies. This syndrome results from direct cellular damage or disparity between the needs and the energy supply to the muscles. We shall return to the pathogenesis of the disturbances of the calcium-phosphorus metabolism in the discussion. ${ }^{17-19}$

\section{Case report}

A 32-year-old man attempted suicide with multiple drugs, including a tricyclic antidepressant, a neuroleptic and a benzodiazopine compound, on 11 February 1992. He was found $14 \mathrm{~h}$ later, in a deep coma, in the left lateral decubitus position. On arrival in the emergency department, rhabdomyolysis of the left upper and lower limbs was diagnosed. The serum CPK reached $10000 \mathrm{IUl}^{-1}$. Renal insufficiency with anuria appeared shortly after. There was peripheral neurological impairment of the left upper and lower limbs, confirmed by electromyography, due to a multiple nerve compression secondary to severe muscular oedema.

The patient was admitted to the rehabilitation department 2 months after a suicide attempt. The oedema persisted but there were signs of a proximal recovery of the neurological deficit. Renal function was normal as was the CPK level and the calcium-phosphorus balance.

Ten days later signs of local inflammation appeared in the left thigh. Plain radiographs were normal, but scintigraphy disclosed intense uptake in the soft tissues around the left hip. Hydroxyprolinuria was slightly elevated, but no other biochemical parameters were abnormal. A diagnosis of heterotopic ossification of the left hip was made, and was visualized on the radiographs a few days later. Computed tomography revealed diffuse atrophy of the left hip, and ossification in the region of the greater trochanter (Figure 1). It was treated by radiotherapy. Six months later, the ossification was stable, and his continued neurological recovery allowed the patient to return home independently. Walking was assisted by means of a calf-sole splint and a walking stick.

\section{Discussion}

The case presented here differs from the usual observations of rhabdomyolysis with acute renal insufficiency; indeed, calcifications associated with rhabdomyolysis are most often observed during the oliguric or anuric phase or on resumption of diuresis. ${ }^{11,12}$ They are often reversible. In this case, the ectopic ossification appeared several weeks after the renal insufficiency had resolved, as was shown by the radiological follow-up. Several factors contributing to heterotopic ossification were present: toxic coma, prolonged immobilisation, metabolic disorders, microtrauma related to the intensive rehabilitation begun shortly before and, of course, the neurological deficit of the involved limb. ${ }^{15,16}$

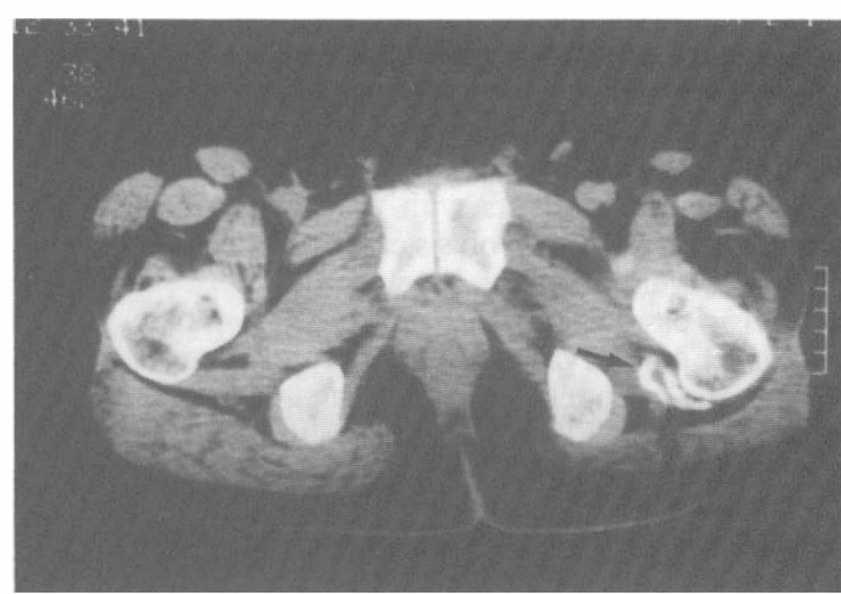

Figure 1 Case 1. Computed tomography: heterotopic ossification of the left hip. No sign of rhabdomyolysis, 2 months after the suicide attempt.

However, this observation leads us to suggest rhabdomyolysis as a possible cause of heterotopic ossification. Indeed, this pathology gives rise to profound alteration in calcium-phosphorus metabolism, especially when it is accompanied by acute renal failure. ${ }^{19}$

Llach et $\mathrm{l}^{18}$ noted six patients with rhabdomyolysis and acute renal insufficiency as follows.

(1) Initially, hypocalcemia with hyperphosphoremia is seen, as well as a decrease in the level of 1,25 $(\mathrm{OH})_{2} \mathrm{D}$ and an elevation in parathormone (PTH). The hypocalcemia might be accounted for by the decreased $1,25(\mathrm{OH})_{2} \mathrm{D}$ and by a bone resistance to the action of $\mathrm{PTH}$, itself secondary to the hyperphosphoremia. It is during this phase that the calcium deposits in the soft tissues are usually demonstrated. Such deposition may be favoured by the administration of calcium, which should be given only if the hypocalcemia is symptomatic. Indeed, as stated by Knochel, ${ }^{17}$ there is a very marked disturbance of the active membrane transport systems of calcium, and of the $\mathrm{Na}+/ \mathrm{K}+$ pump, in the tissues affected by rhabdomyolysis. Calcium accumulates in the sarcoplasm, which may explain the hypocalcemia and the calcium deposition, and aggravates the cellular injuries. $\mathrm{Akmal}^{20}$ reported that initial hypocalcemia may be seen even in the absence of renal insuffuciency.

(2) During the polyuric phase, hypercalcemia with increaed levels of $1,25(\mathrm{OH}) 2 \mathrm{D}$ and PTH is seen. This hypercalcemia could be secondary to a mobilisation of calcium of the soft tissues and the bone, to dehydration and elevated PTH. At this stage soft tissues calcifications often disappear. However, conversely marked and prolonged hypercalcemia may induce calcifications.

The pathogenesis of the disturbances of the phosphorus-calcium metabolism in rhabdomyolysis remains to be elucidated. However, it is recognised that 
rhabdomyolysis favours the intracellular accumulation of calcium in the affected muscles. Chantraine and Minaire $^{2}$ have reviewed the role of bone calcium turnover disturbances in the sublesional zones, with disturbances of calcitonin and PTH in the heterotopic ossifications of patients with paraplegia. It therefore appears that rhabdomyolysis creates conditions favouring the development of heterotopic ossification, particularly if associated with a neurological deficit.

A second observation seems to confirm our hypothesis: in one of our female patients who had become paraplegic from T11 level as the result of a car accident, computed tomography revealed signs of rhabdomyolysis of the iliopsoas and the right internal obturator muscles 2 weeks before the appearance of heterotopic ossification of the right hip (Figure 2).

But histological data should be consistent with this hypothesis, because heterotopic ossification and rhabdomyolysis share common aetiologies (for instance trauma and toxic coma) and may consequently coexist, without any causal relationship.

Soft tissue calcification in rhabdomyolysis is due to calcium salt deposition in injured soft tissues. ${ }^{10,12,19}$ Heterotopic ossification consists in bone metaplasia of the connective tissue, preceded by inflammatory reaction with cellular infiltration. ${ }^{2}$ There may be a continuum between calcium deposition, osteoid and lamellar bone formation. Repeated pathological examinations would allow us to confirm this supposition, but unfortunately they were not performed on our two patients.

Rhabdomyolysis is often underappreciated, especially when it affects a limited number of muscles. It would be of interest to perform more systematic investigations in those with spinal lesions (CPK determination and imaging techniques) to know whether it precedes the development of heterotopic ossification. One could thus identify patients at risk of heterotopic ossification, who would benefit from a more systematic follow-up, improving the management of the ossifications at an early stage and thereby limiting their consequences. In these patients at risk, other aetio-

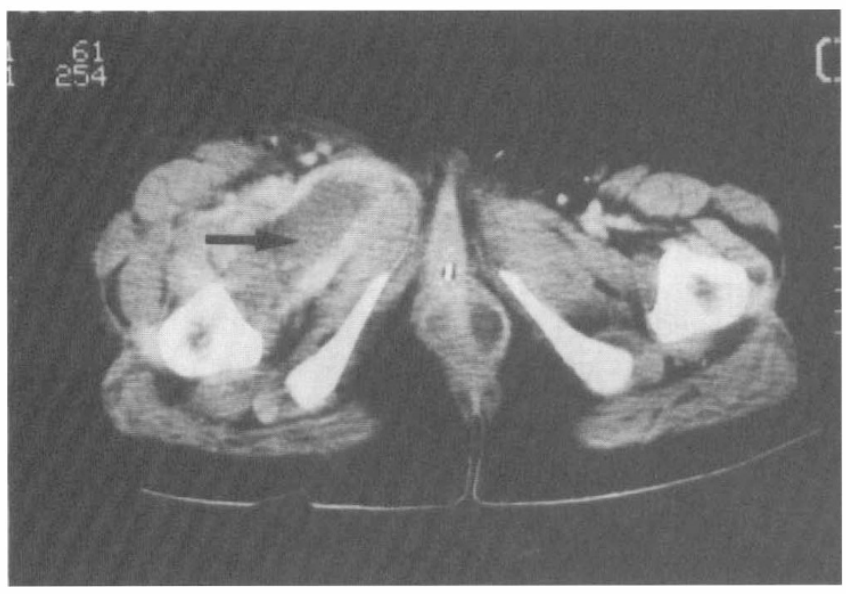

Figure 2 Case 2. Computed tomography: oedematous infiltration of the right thigh. logical factors could also be limited, such as microtrauma associated with rehabilitation.

\section{Conclusion}

Rhabdomyolysis should be considered as a possible cause of heterotopic ossification. This may lead to progress in the understanding of the pathophysiological mechanisms and improve preventative measures or result in earlier diagnosis and better management. Further study and more detailed investigations, pathological correlations, determination of calcium kinetics and hormone determinations are recommended.

\section{References}

1 Dejerine J, Ceillier A. Paraosteoarthropathies des paraplégiques par lésion médullaire. Ann Med 1919; 5: 497-535.

2 Chantraine A, Minaire P. Paraosteoarthropathies. A new theory and mode of treatment. Scand J Rehabil Med 1981; 13: 31-37.

3 Minaire P. Les paraosteoarthropathies neurogénes. Ann Med Phys 1982; 25: 51-55.

4 Freed J, Hahn H, Menter R, Dillon T. The use of three phase bone scan in the early diagnosis of heterotopic ossification and in the evaluation of Didronel therapy. Paraplegia 1982; 20: 208-216.

5 Bodley R, Jamous A, Short D. Ultrasound in the early diagnosis of heterotopic ossification in patients with spinal injuries. Paraplegia 1993; 31: 500-506.

6 Kewalramani LS. Ectopic ossification. Am J Phys Med 1977; 56: $99-121$.

7 Michelsson JE, Granroth G, Andersson LC. Myositis ossificans following forcible manipulation of the leg. J Bone Joint Surg Am 1980; 62: 811-815.

8 Daud O, Sett P, Burr RG, Silver JR. The relationship of heterotopic ossification to passive movements in paraplegic patients. Disability and Rehabilitation 1993; 15: 114-118.

9 Minaire P, Betuel H, Girard R, Pilonchery G. Neurologic injuries, paraosteoarthropathies, and human leucocyte antigens. Arch Phys Med Rehabil 1980; 61: 214-215.

10 Perrot D, Mottin J. Rhabdomyolyses traumatiques et non traumatiques. In: Encycl Med Chir 1985 (Paris, France) Anesthésie Réanimation, 36918 A10, 10 p.

11 George $\mathrm{C}$ et al. Muscle calcification in rhabdomyolysis. Detection with Technetium pyrophosphate. Nouv Presse Med 1979; 8: $3365-3366$.

12 Towers MJ, Downey DB, Poon PY. Psoas muscle calcification and acute renal failure associated with nontraumatic rhabdomyolysis: computed tomography features. J Comput Assist Tomogr 1990; 14: 1027-1029.

13 Kaplan GN. Ultrasonic appearance of rhabdomyolysis. Am J Roentgenol 1980; 134: 375-377.

14 Barloon TJ, Zachar CK, Harkens KL, Honda H. Rhabdomyolysis: computed tomography findings. J Comput Assist Tomogr 12: $193-195$.

15 Frymoyer PA, Giammarco R, Farrar FM, Schroeder ET. Tecnetium TC-99m Medronate bone scanning in rhabdomyolysis. Arch Intern Med 1985; 145: 1991-1995.

16 Patel R, Mishkin FS. Tecnetium pyrophosphate imaging in acute renal failure with nontraumatic rhabdomyolysis. Am J Roentgenol 1986; 147: 815-817.

17 Knochel JP. Serum calcium derangements in rhabdomyolysis. N Engl J Med 1981; 305: 161-162.

18 Llach F, Felsenfeld AJ, Haussler MR. The physiopathology of altered calcium metabolism in rhabdomyolysis-induced acute renal failure. $N$ Engl J Med 1981; 305: 117-123.

19 Poels PJE, Gabreëls FJM. Rhabdomyolysis: a review of the literature. Clin Neurol Neurosurg 1993; 95: 175-192.

20 Akmal $\mathrm{M}$ et al. Resolution of muscle calcification in rhabdomyolysis and acute renal failure. Ann Intern Med 1978; 89: 928-930. 